\title{
Preparation of Polyimide Films Derived from a Novel Precursor Pre-imidized Poly (amic acid) Ammonium Salt
}

\author{
Liu-Cheng $\mathrm{HE}^{1, \mathrm{a}}$, Bai-Qing TANG ${ }^{1, \mathrm{~b}}$, Li-Xing DAI ${ }^{1, \mathrm{c},{ }^{*}}$ \\ ${ }^{1}$ College of Chemistry, Chemical Engineering and Materials Science, Soochow University, Suzhou, \\ Jiangsu, 215123, People's Republic of China \\ aheliucheng0818@163.com, tangbaiqing15@163.com, ${ }^{\mathrm{c}}$ dailixing@suda.edu.cn \\ ${ }^{*}$ Corresponding author
}

Keywords: Poly (amic acid) Ammonium Salt, Pre-imidization, Hydrolytic Stability, Chemical Structure, Property.

\begin{abstract}
In this paper, a series of polyimide films were prepares through a novel precursor pre-imidized poly (amic acid) ammonium salt (PI-PAAS). The chemical structure of PI-PAAS films was confirmed by $1 \mathrm{H}$ NMR, and the degree of pre-imidization was confirmed by FT-IR spectra. And the intrinsic viscosity of the PI-PAAS solution was not distinctly changed after 90 days, while the viscosity of poly (amic acid) (PAA) obviously decreased with storage time. It was suggested that hydrolytic stability of PI-PAAS was significantly improved as compared with that of PAA due to the shielded carboxyl groups on PI-PAAS, which prevented the molecular chain of the polymer from decomposition. Moreover, PI films derived from PI-PAAS precursors possessed superior mechanical properties.
\end{abstract}

\section{Introduction}

Aromatic polyimides (PIs), as a kind of high-performance polymer, are well-known for their high thermal stability, excellent mechanical properties, outstanding dimensional stability and chemical resistance [1]. Because of these outstanding properties, the applications of fibers, films, coating, and composites have been studied for many years [2]. However, these polymeric materials have the disadvantage of poor solubility and high glass transition temperature because of the rigid backbone structures, making them extremely difficult to process. So, these drawbacks have been confined the investigation of polyimide precursor polymers, for example, poly (amic acid) (PAA) [3], poly (amic acid) ammonium salts (PAAS) [4] and so on.

In general, the conventional preparation of PI included two steps [5]: firstly, PAA was derived from the reaction of diamines and dianhydrides monomers in polar solvent, such as N,N-dimethylformamide (DMF), dimethyl sulfoxide (DMSO), and N,N-dimethylacetamide (DMAc). Secondly, the above PAA solution was used to make films [6], coatings [7] or fibers [8], and then the complete cyclization of the above materials was finished by the thermal or chemical imidization. The conversion from PAA to the polyimide (PI) was most commonly accomplished by the thermal treatment of PAA in the thermal imidization furnace.

However, some disadvantages were showed on the traditional preparation process [9], such as: high thermal imidization temperature and low hydrolytic stability of PAA precursors. To overcome these drawbacks, more and more researchers have paid more attention to PAAS, which can act as the intermediate of polyimide. For example, Facinelli et al. [10] studied a series of PAAS precursors to obviously enhance the water solubility by neutralizing the poly (amic acid)'s carboxylic acid groups with a suitable organic base. These water-soluble PAAS precursors provided an environmentally friendly method for preparing PI. In our previous work [11], the synthesis and characterization of PI film based on stable PAAS precursors has been reported. It was found that the hydrolytic stability and water solubility of PAAS precursors could be significantly enhanced by neutralizing the poly (amic acid)'s carboxylic acid groups with triethylamine (TEA). In addition, Ding et al. [12] found that PAAS precursors could be quantitatively imidized at $150{ }^{\circ} \mathrm{C}$, the imidization temperature of PAAS 
was much lower than that of PAA. The preparation method of PI film derived from PAAS precursors overcame those above disadvantages, but the mechanical and thermal properties of PI films were decreased concomitantly [9].

In this paper, it is expected that through combining the processes of pre-imidization and salifying, carboxyl groups on PAA can be fully shield without affecting its flowability, so the related properties of PAA and its derivative can be improved. A partially pre-imidization poly (amic acid) ammonium salt (PI-PAAS) was prepared and PI films were derived from it. The structure of PI-PAAS was confirmed using ${ }^{1} \mathrm{H}$ NMR. Hydrolytic stability of PI-PAAS was studied through the characterization of intrinsic viscosities, and the mechanical properties of final PI films were also investigated.

\section{Experimental}

\section{Materials}

Pyromellitic dianhydride (PMDA), 4,4'-oxydianiline (ODA), p-phenylenediamine (PDA), acetic anhydride $\left(\mathrm{Ac}_{2} \mathrm{O}\right)$, pyridine (Py), triethylamine (TEA), and $\mathrm{N}, \mathrm{N}$-dimethylacetamide (DMAc) were purchased from Sinopharm Chemical Reagent Co., Ltd. PMDA was dried in vacuum at $120^{\circ} \mathrm{C}$ for 24 $\mathrm{h}$ to ensure complete anhydride cyclization prior to use. ODA and PDA were purified by vacuum sublimation prior to use. TEA was analytical grade and used without any further purifying.

\section{Preparation of PAA and PI-PAAS Solutions}

The PAA and PI-PAAS solutions were synthesized by the proposed reaction mechanisms as shown in Fig. 1. A representative polymerization was as follows: an appropriate amount of ODA and PDA (molar ratio of PDA/ODA=2:8) was added into DMAc in a three-necked flask equipped with a mechanical stirrer and nitrogen inlet at $0 \pm 1{ }^{\circ} \mathrm{C}$. After ODA and PDA were completely dissolved in DMAc, an equimolar amount of PMDA was divided into three groups, and each was added to the solution with a time interval of $0.5 \mathrm{~h}$. When PMDA was completely dissolved, the solution in the flask reacted for $2 \mathrm{~h}$ to form homogeneous and viscous PAA solution which contained a $9.5 \mathrm{wt} \%$ total solid. PI-PAAS solutions were synthesized by adding different amounts of TEA 80, 60, 40, 20 and 0 mol\% relative to original amino groups of PAA into the PAA solution mentioned above. After reacted for $4 \mathrm{~h}$ at $0 \pm 1{ }^{\circ} \mathrm{C}$, poly (amic acid) ammonium salt (PAA-PAAS) solutions containing different content of TEA were obtained. Then different amount of dehydration reagents $\mathrm{Ac}_{2} \mathrm{O}$ and $\mathrm{Py}$ of 20, 40, 60, 80 and $100 \mathrm{~mol} \%$ (molar ratio of $\mathrm{Ac}_{2} \mathrm{O} / \mathrm{Py}=1: 1$ ) relative to original amino groups of the PAA was added into the PAA-PAAS solutions containing TEA 80, 60, 40, 20 and 0 mol\%, respectively. After reacting for 3 $\mathrm{h}$, PI-PAAS solutions with different pre-imidization degree corresponding to the different amount of dehydration reagents were obtained, and samples of PI-PAAS were abbreviated as PI-20-PAAS-80, PI-40-PAAS-60, PI-60-PAAS-40, PI-80-PAAS-20, and PI-100, respectively.

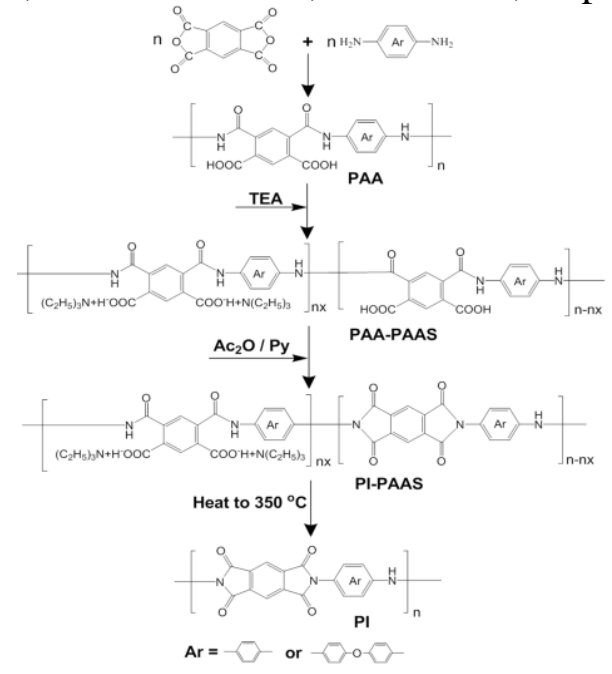

Fig. 1 Synthesis of polyimide via the PI-PAAS precursor 


\section{Preparation of PAA, PI-PAAS, PAAS Films and the Corresponding PI Films}

The PAA and PI-PAAS films about $20 \sim 30 \mu \mathrm{m}$ thick with different pre-imidization degree were obtained by casting PAA and PI-PAAS solutions onto glass plates and then dried at $60{ }^{\circ} \mathrm{C}$ in a vacuum oven for $24 \mathrm{~h}$ to eliminate DMAc. The final PI films were obtained by removing PAA and PI-PAAS films from the glass plates after they were thermally baked in a vacuum oven at $100^{\circ} \mathrm{C}$ for $1 \mathrm{~h}, 150{ }^{\circ} \mathrm{C}$ for $1 \mathrm{~h}, 200{ }^{\circ} \mathrm{C}$ for $1 \mathrm{~h}, 250^{\circ} \mathrm{C}$ for $1 \mathrm{~h}, 300^{\circ} \mathrm{C}$ for $0.5 \mathrm{~h}, 350{ }^{\circ} \mathrm{C}$ for $0.5 \mathrm{~h}$, successively. The final PI films derived from PAA, PI-60-PAAS-20, PI-40-PAAS-60 and PI-20-PAAS-80 films were marked as $\mathrm{PI}_{\mathrm{a}}, \mathrm{PI}_{\mathrm{i}-60}, \mathrm{PI}_{\mathrm{i}-40}$ and $\mathrm{PI}_{\mathrm{i}-20}$, respectively. Unfortunately, PI-100 and PI-80-PAAS-20 films cannot be prepared because of their very high solution viscosity.

\section{Characterization}

${ }^{1} \mathrm{H}$ NMR spectra was recorded at room temperature on a Unity Inovr 400 instrument with dimethylsulfoxide- $\mathrm{d}_{6}\left(\right.$ DMSO- $\left.\mathrm{d}_{6}\right)$ as solvent and tetramethylsilane (TMS) as reference, which was used to confirm the chemical structure of PI-PAAS. Fourier transform infrared (FT-IR) spectra were recorded on a Nicolet 5700 Fourier transform spectrophotometer, the scanning range was $4000 \sim 400 \mathrm{~cm}^{-1}$. The pre-imidization degree (ID) of dried PI-PAAS sample was determined according to the reported method [13], in which the band of $1380 \mathrm{~cm}^{-1}$ was selected for quantifying ID, and the aromatic band at $1500 \mathrm{~cm}^{-1}$ (C-C stretching of the p-substituted benzene back-bone) was selected as the internal standard. The intrinsic viscosities of the PAA and PI-PAAS solutions were measured with an Ubbelohde viscometer by using DMAc as solvent at $30{ }^{\circ} \mathrm{C}$. The standard concentration $\left(\mathrm{C}_{\mathrm{s}}\right)$ was $1 \mathrm{~g} / \mathrm{dl}$. In order to minimize the effect of polyelectrolyte on the viscosity measurement, DMAc was purified by distilling over phosphorus pentoxide $\left(\mathrm{P}_{2} \mathrm{O}_{5}\right)$ under reduced pressure. And all the DMAc solutions were filtered through $0.5 \mu \mathrm{m}$ millipore filters before measurement. Then the intrinsic viscosity $[\eta]$ was determined from the intercept extrapolated at $C_{p}=0$ in both plots of $\eta_{\mathrm{sp}} / \mathrm{C}_{\mathrm{p}}$, vs. $\mathrm{C}_{\mathrm{p}}$, and of $\ln \eta_{\mathrm{rel}} / \mathrm{C}_{\mathrm{p}}$ vs. $\mathrm{C}_{\mathrm{p}}$, where $\eta_{\mathrm{sp}}$ and $\eta_{\mathrm{reI}}$ were specific and relative viscosities, respectively [14]. Mechanical tensile properties of PI films were performed at room temperature on an YG004N electromechanical universal testing machine. Samples of PI film were cut into $5 \mathrm{~mm} \sim 10 \mathrm{~mm}$ strips along the film casting direction. Average values of more than five measurements were taken for each sample.

\section{Results and Discussion}

\section{Determination of the Chemical Structure of PI-PAAS}

The chemical structure of PI-60-PAAS-40, as a typical sample of PI-PAAS, is characterized by ${ }^{1} \mathrm{H}$ NMR. As shown in Fig. 2 (A), two ethyl protons of TEA which are represented as $-\mathrm{CH}_{3}$ and $-\mathrm{CH}_{2}$, which are identified at 0.95 (peak a) and 2.44 (peak b) ppm, while those in PI-60-PAAS-40 are shifted to 1.11 and $2.91 \mathrm{ppm}$ as shown in Fig. 2 (C), respectively. As indicated by Echigo Y [15], the obvious chemical shifts are caused by the shielding effect of $-\mathrm{COO}^{-} \mathrm{H}^{+}$proton in poly (amic acid) ammonium salt of PI-60-PAAS-40. In addition, an obvious peak (g) at $10.59 \mathrm{ppm}$ of carboxyl of PAA appears which is shown in Fig. 2 (B), while the peak do not appear in PI-PAAS as shown in Fig. 2 (C), suggesting no carboxyl existing. The chemical structure of PI-PAAS with different pre-imidization degree is also characterized by FT-IR spectroscopy.

Fig. 3 (A) shows the FT-IR spectrum of PAA and PI-PAAS with different pre-imidization degree precursors. It can be seen that the intensity of absorbance bands of PAA $\left(1655 \mathrm{~cm}^{-1}\right.$, carbonyl in -COOH, and $3300 \mathrm{~cm}^{-1}$, hydroxyl in -COOH (not shown)) decreases gradually with the increase of pre-imidization from 0 to $60 \%$. Meanwhile, the characteristic absorbance bands of imide ring (1780, 1724 and $1380 \mathrm{~cm}^{-1}$ ) increase obviously. In addition to imide ring, isoimide is also usually formed in the chemical imidization process of PAA. However, the characteristic absorbance band of isoimide (1800 $\mathrm{cm}^{-1}$ ) is barely detectable in FT-IR spectrum of those partially imidized samples. Thus, the main copolymer marked PI-PAAS is obtained from the chemical imidization process, and isoimide could be ignored. Moreover, Fig. 3 (B) summarizes the pre-imidization calculated from FT-IR spectrum, in which 
it can be seen that all the experimental data are in good agreement with the theoretical ones, indicating it is a successful method to control the pre-imidization by controlling the amount of dehydrating reagents. Thus, the partially pre-imidization process is useful to obtain the PI-PAAS copolymers.

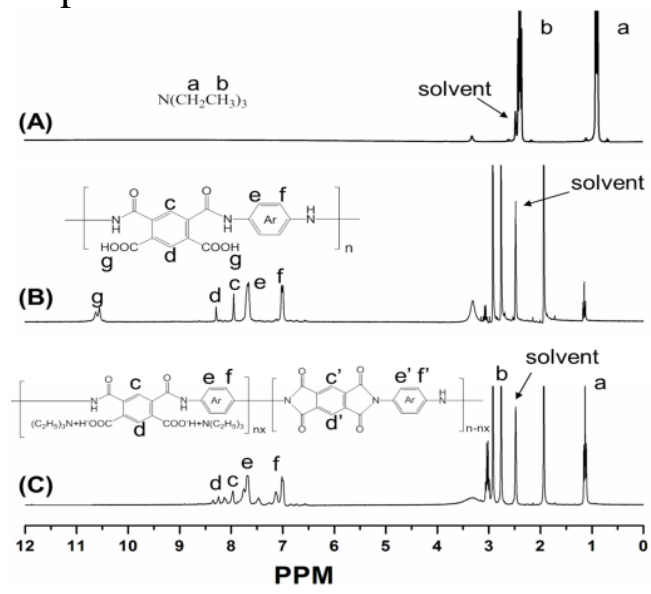

Fig. $2{ }^{1} \mathrm{H}$ NMR (DMSO-d ${ }_{6}$ ) characterization of TEA (A), PAA (B), and PI-PAAS (C)
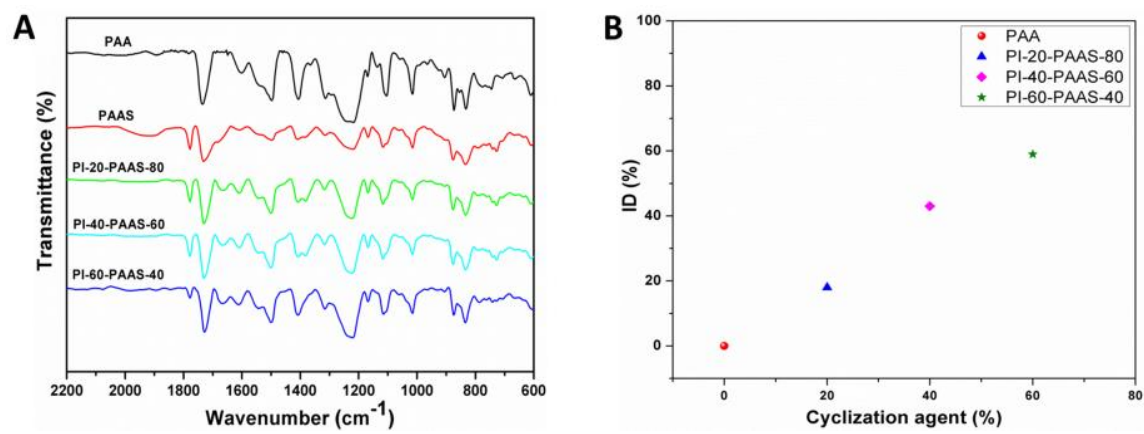

Fig. 3 (A) The FT-IR spectrum of PAA and PI-PAAS with different pre-imidization degree precursors. (B) The experimental imidization degree of PI-PAAS

\section{Solution Properties of PI-PAAS Precursors}
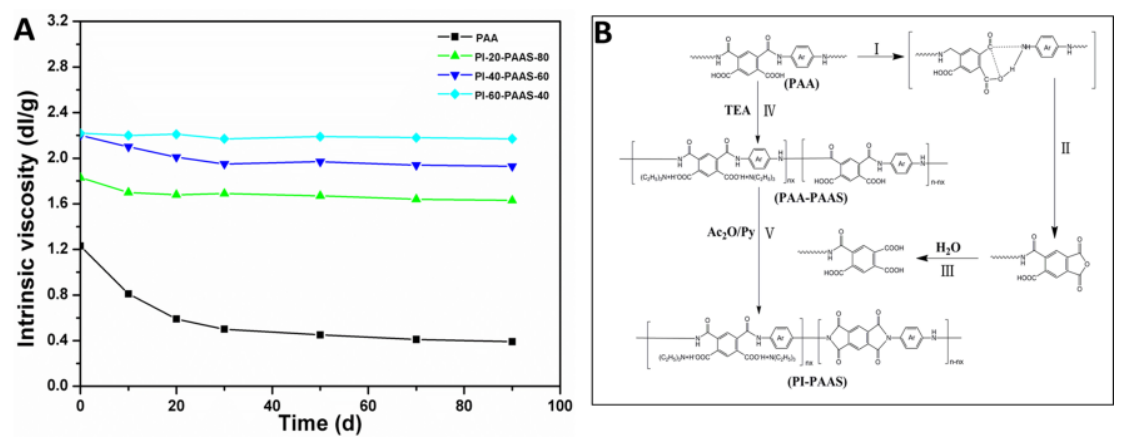

Fig. 4 (A) The intrinsic viscosities of PAA and PI-PAAS solutions with storage time under room temperature. (B) The degradation mechanism of PAA (I, II, III) and the scheme of preparation for PI-PAAS (IV, V)

The intrinsic viscosities [ $\eta]$ of PAA and PI-PAAS with different pre-imidization degree are shown in Fig. 4 (A). At storage time of 0 day, with the increase of amount of dehydration reagents from 20 mol\% (PI-20-PAAS-80) to $60 \mathrm{~mol} \%$ (PI-60-PAAS-40), or the decrease of TEA contents from 80 $\mathrm{mol} \%$ to $40 \mathrm{~mol} \%$, that is, the increase of pre-imidization degree or the decrease of salifying degree, [ $\eta$ ] of PI-PAAS increases from 1.70 to $2.13 \mathrm{dl} / \mathrm{g}$, which are distinctly higher than that of PAA (1.23 $\mathrm{dl} / \mathrm{g}$ ). In addition, the $[\eta]$ of PI-PAAS solutions is not changed distinctly after 90 days, particularly for the PI-60-PAAS-40 sample with higher pre-imidization degree. It is suggested that although both 
pre-imidization and salifying can shield carboxyl on PAA, pre-imidization is good for increasing or stabilizing the molecular weight compared with salifying. However, [ $\eta]$ of PAA decreases continuously with storage time which is caused by the acid-catalyzed hydrolysis [16], and the decrease is particularly evident during the first 5 days. Just as shown in Fig. 4 (B) (I, II, III), the PAA is degraded into the amide by the acid-catalyzed hydrolysis, which involves intramolecular cyclization of the carboxyl groups onto the amide linkage with the elimination of amine. However when PAA is converted to PI-PAAS as shown in Fig. 4 (B) (IV, V), the reaction of acid-catalyzed hydrolysis is effectively suppressed due to the absence of carboxyl.

\section{Mechanical Properties of PAA and PI-PAAS Films with Different Pre-imidization Degree}

The changes of mechanical properties of PAA and PI-PAAS with different pre-imidization degree films with storage time at room temperature are shown in Fig. 5, respectively. It could be seen that both the tensile strength and the elongation of PAA films obviously decrease with storage time during 30 days, but the mechanical properties of PI-PAAS are not decreased with storage time, and gradually enhance with the increase of pre-imidization degree, indicating that the PI-PAAS could effectively suppress the acid-catalyzed hydrolysis.
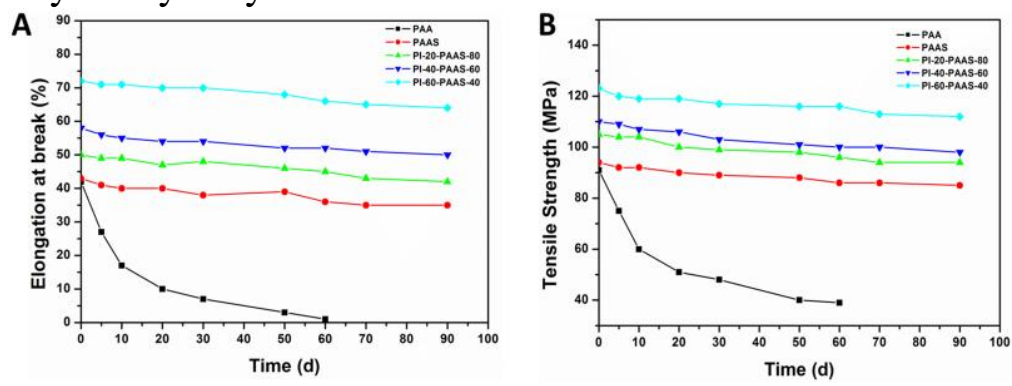

Fig. 5 Decay of tensile strength and elongation for PAA, PI-PAAS and PAAS films with storage time under room temperature

\section{Mechanical Properties of Final PI Films}

Fig. 6 shows the mechanical properties of different PI films. It can be seen that the mechanical properties of $\mathrm{PI}_{\mathrm{i}}$ are all higher than $\mathrm{PI}_{\mathrm{a}}$. As was stated above, PAA degraded because of its low hydrolytic stability before it converts to $\mathrm{PI}_{\mathrm{a}}$, leading to decreasing the mechanical properties. And the mechanical properties of $\mathrm{PI}_{\mathrm{i}}$ are gradually enhanced with the increase of pre-imidization degree. On one hand, PI-PAAS with higher pre-imidization degree has higher [ $\eta]$ (molecular weight), which is more stable. On the other hand, it is deduced that through pre-imidization the rigid PI chain segments has high mobility in solution, while the chains with higher rigidity pack more regularly in solution, leading to increasing the degree of crystallinity. So, the PI films have the increasing mechanical properties due to the salifying and pre-imidization.

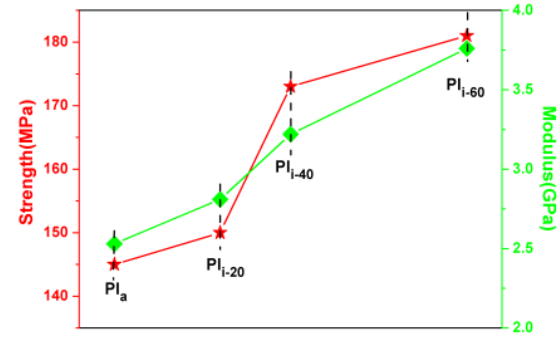

Fig. 6 The mechanical properties of $\mathrm{PI}_{\mathrm{a}}$ and $\mathrm{PI}_{\mathrm{i}}$ films with di fferent pre-imidization degree

\section{Summary}

In this study, series of polyimide films were prepared through PI-PAAS with different pre-imidization degree. Through pre-imidization and salifying, all carboxyls on PAA were shielded, but the PI-PAAS solutions remained good flowability and processibility. No distinct changes were observed in the 
intrinsic viscosity of the PI-PAAS after 90 days, while the intrinsic viscosity of PAA obviously decreased continuously with storage time. The results suggested that hydrolytic stability of the PI-PAAS films was significantly improved as compared with that of PAA film due to the PI-PAAS shielded carboxyl groups. Moreover, PI films prepared from PI-PAAS precursors possessed superior mechanical properties.

\section{Acknowledgement}

This research was supported by Natural Science Fund of Jiangsu Province (BK2012623) and a Project Funded by the Priority Academic Program Development of Jiangsu Higher Education Institutions.

\section{References}

[1]M. X. Ding, Polyimides: chemistry, relationship between structure and properties and materials, Beijing: Chinese Science Press. 2006.

[2]D. J. Liaw, P. N. Hsu, W. H. Chen, S. L. Lin, High glass transitions of new polyamides polyimides, and poly (amide-imide)s containing a diphenylamine group: synthesis and characterization, Macromolecules. 35 (2002) 4669-4676.

[3]K. P. Pramoda, T. S. Chung, S. L. Liu, H. Oikawa, A. Yamaguchi, Characterization and thermal degradation of polyimide and polyamide liquid crystalline polymers, Polymer degradation and stability. 92 (2007) 1265-1278.

[4]Y. Ding, B. Bikson, J. K. Nelson, EPA Patent 1,086,737. (2001)

[5]P. R. Buch, A. V. R. Reddy, Synthesis, characterization and thermal properties of soluble aromatic poly(amide imide)s, Polymer. 46 (2005) 5524-5532.

[6]S. B. Mehtap, B. Ismail, A. G. Mehmet, Structural characterization of silica modified polyimide membranes, Polymers for advanced technologies. 17 (2006) 6-11.

[7]J. H. Dai, M. S. Daniel, L. B. Merlin, Ultrathin, layered polyamide and polyimide coatings on aluminum, Industrial \& engineering chemistry research. 39 (2000) 3528-3535.

[8]Q. H. Zhang, M. Dai, M. X. Ding, D. J. Chen, L. X. Gao, Mechanical properties of BPDA-ODA polyimide fibers, European polymer journal. 40 (2004) 2487-2493.

[9]J. H. Chang, M. J. Chen, R. J. Farris, Effect of heat treatment on the thermal and mechanical properties of a precursor polymer: polyhydroxyamide, Polymer. 39 (1998) 5649-5654.

[10]J. V. Facinelli, S. L. Gardner, L. Dong, C. L. Sensenich, R. M. Davis, J. S. Riffle, Controlled molecular weight polyimides from poly (amic acid) salt precursors, Macromolecules. 29 (1996) 7342-7350.

[11]D. D. Cai, J. F. Su, M. Huang, Y. H. Liu, J. J. Wang, L. X. Dai, Synthesis, characterization and hydrolytic stability of poly (amic acid) ammonium salt, Polymer degradation and stability. 96 (2011) 2174-2180.

[12]Y. Ding, B. Bikson, J. K. Nelson, Polyimide membranes derived from poly (amic acid) salt precursor polymers. 1. Synthesis and characterization, Macromolecules. 35 (2002) 905-911.

[13]M. Marek, P. Schmidt, B. Schneider, M. Marek, Imidization of polypromellitamic acid based on 4, 4'-methylenedianiline, Die makromolekulare chemie. 191 (1990) 2631-2637.

[14]S. I. Kim, T. J. Shin, S. M. Pyo, J. M. Moon, M. Ree, Structure and properties of rodlike poly (p-phenylene pyromellitimide)s containing short side groups, Polymer. 40 (1999) 1603-1610.

[15]Y. Echigo, N. Miki, I. Tomioka, Preparation of poly (bis-(trialkylammonium)-oxydiphenylenepyromellitamate) films: a useful polyimide precursor film, Journal of applied polymer science. 35 (1997) 2493-2499.

[16]Y. J. Kim, T. E. Glass, G. D. Lyle, J. E. McGrath, Kinetic and mechanistic investigations of the formation of polyimides under homogeneous conditions, Macromolecules. 26 (1993) 1344-1358. 